\title{
De l'intérêt des cheveux et de la spectrométrie de masse tandem pour la soumission chimique : à propos de neuf cas
}

\section{Interest of hair and tandem mass spectrome- try for chemical submission : about 9 cases}

Gilbert PÉPIN, Marjorie CHEZE, Gaëlle DUFFORT, François VAYSSETTE

Laboratoire d'Expertises TOXLAB, 7, rue Jacques Cartier - 75018 PARIS Tél : 0158592800 - Fax : 0158592801 - e-mail : labtoxlab@aol.com

(Reçu le 10 octobre 2002 ; accepté le 25 octobre 2002)

\section{RÉSUMÉ}

Les auteurs présentent les dernières avancées analytiques et médico-légales pour la mise en évidence de soumission chimique dans le cadre d'expertise judiciaire médico-légale. Ces actes criminels médiatisés actuellement en expansion, sont illustrés par neuf exemples difficiles que nous avons pu solutionner. Ces cas ont nécessité la mise en ouvre de techniques particulièrement spécifiques et sensibles telles que la chromatographie liquide haute performance couplée à la spectrométrie de masse tandem ou la chromatographie gazeuse couplée à la spectrométrie de masse tandem. Elles sont pratiquées notamment pour l'analyse des phanères, seul milieu utilisable lorsque la victime porte plainte plusieurs jours après les faits et lorsque le xénobiotique en cause possède une fenêtre de détection très faible dans les fluides biologiques. Les cheveux présentent également l'intérêt de pouvoir matérialiser l'abstinence à la substance, administrée à l'insu, avant et après les faits.

\section{SUMMARY}

The authors present the last analytical and forensic developments for revealing chemical submission within the framework of forensic judicial expertise. These criminal acts witch are given a lot of media coverage are, at present, increasing and are here documented by 9 difficult examples. These cases required specific and sensitive techniques such as high performance liquid chromatography - tandem mass spectrometry and gas chromatography-tandem mass spectrometry. They are notably practised for the analysis of hair, a very useful sample when the victim raises a complaint only several days after the facts and when the drug involved has a very weak window of detection in biological fuids. Hair analysis enables to show the abstinence in drug, administered without victim's knowledge, before and after the facts.

These techniques should allow the toxicologist to bring the definitive scientific proof, usefull for the authorities' applicants to confuse the offender. In this context of exceptional 
Ces techniques doivent permettre au toxicologue d'apporter la preuve scientifique irréfutable nécessaire aux autorités requérantes pour confondre la personne mise en cause.

Dans ce contexte de performance analytique exceptionnelle, il nous semblerait logique et utile à la manifestation de la vérité, pour ces faits criminels, à l'image de ce qui a été réalisé pour les empreintes génétiques dans le domaine judiciaire, que le Ministère de la Justice en liaison avec le Ministère de la Santé agréent au niveau national quelques laboratoires spécialisés, très bien équipés ayant démontré leur compétence par des épreuves qualifiantes pour la recherche des substances de la soumission chimique dans le sang, les urines et surtout les phanères.

\section{MOTS-CLÉS}

Soumission chimique, viols, vols, cheveux, sang, urine, atropine, scopolamine, LSD, GHB, funitrazépam, clonazépam, niaprazine, zopiclone.

\section{Introduction}

La soumission chimique consiste à administrer à l'insu d'une personne, une substance psychoactive à des fins criminelles visant à réduire sa volonté, son libre arbitre, son indépendance ou toute autre forme de pensée d'émancipation physique ou psychique de la victime. Celle-ci peut revêtir des formes multiples. Nous n'aborderons pas les toxiques les plus couramment employés tels l'alcool et le cannabis (1), mais des cas plus difficiles où le GHB, le LSD, le flunitrazépam, le clonazépam et une plante incapacitante, le Datura, ont été identifiés et mis en cause.

La fréquence des cas de soumission chimique est largement discutée $(2,3)$, certains affirmant qu'elle est surestimée, d'autres qu'elle est sous-évaluée.

Frank Questel et collaborateurs nous apprennent que les Urgences Médico-Judiciaires (UMJ) de l'Hôtel Dieu à Paris ont recensé, sur 4 ans, 82 dossiers de soumission médicamenteuse (4). Un autre article publié plus récemment (5) dans la même revue par J.S. Raul nous indique que le service d'UMJ de Strasbourg a recensé 30 dossiers de soumission médicamenteuse sur 127 dossiers d'agression sexuelle en 18 mois (du $1^{\text {er }}$ janvier 2000 à la mi-juillet 2001).

La mise en évidence de la soumission chimique est toujours extrêmement délicate à réaliser et peut s'appuyer entre autres éléments sur une preuve scientifique irréfutable issue des examens toxicologiques effectués sur les prélèvements biologiques de la victime; en effet, dans les cas de viols où la relation sexuelle est bien établie, l'auteur des faits déclare régulièrement que la victime était bien évidemment consentante. L'obtention de cette preuve scientifique irréfutable est rendue très difficile car le délai écoulé entre les faits et les prélèvements biologiques (sang, urine) est souvent trop long analytical performance, it seems to us logical and useful for the demonstration of the truth, according to what was realized for genetic fingerprints in the judicial domain, that the Ministry of Justice in contact with the Ministry of Health approve at the national level some specialized, very well equipped laboratories and having unsettled their competence by qualifying tests in the research for substances in the blood, the urine and especially hair in case of chemical submission.

\section{KEY-WORDS}

Chemical submission, rapes, robbery, hair, blood, urine, atropine, scopolamine, LSD, GHB, flunitrazepam, clonazepam, niaprazine, zopiclone.

(6) pour permettre la détection des agents de soumission chimique dont la demi-vie est souvent très courte $(7,8)$.

C'est pourquoi le prélèvement orienté et l'analyse de cheveux sont particulièrement importants, car ils peuvent apporter une preuve probante à posteriori (9).

L'expérience nous a appris que le prélèvement de cheveux doit être effectué deux à trois semaines minimum après la date des faits pour que, par exemple dans le cas du $\gamma \mathrm{OH}$, la partie du cheveu proche du cuir chevelu ne soit pas contaminée par la sueur dans laquelle se retrouve également l'agent de soumission chimique à une concentration supérieure à celle du cheveu.

Cependant, les caractéristiques spécifiques du cheveu (10) présentent dans la soumission chimique plusieurs avantages particulièrement intéressants par rapport aux autres milieux biologiques :

$\left.1^{\circ}\right)$ La grande majorité des molécules exogènes incluses dans la kératine sont protégées de toute dégradation qu'elle soit chimique, physique, enzymatique ou bactériologique.

$2^{\circ}$ ) Les cheveux poussant en moyenne de 1 centimètre par mois, le tronçonnage de la mèche permettra de dater la prise de l'agent de soumission par rapport à la date des faits.

$3^{\circ}$ ) L'analyse en dehors de la période des faits (avant et après les faits), permettra éventuellement de matérialiser l'abstinence de la victime à l'agent de soumission chimique et apportera donc la preuve tant recherchée de l'administration de la substance au moment des faits. Le cas du GHB, présent de façon endogène est particulier, seule une variation du taux au moment des faits sera probante et permettra une interprétation du résultat. (6-7,11-13). Pour les raisons précédemment indiquées, il est capital d'effectuer le prélèvement 
orienté environ trois semaines après les faits. $(11,14)$. La soumission chimique dans le cadre du viol est un acte criminel qui a été largement médiatisé depuis quelques années ; citons quelques articles relevés dans la presse : «Pétition contre une benzodiazépine hypnotique détoumée de son usage», Le Quotidien du médecin 30/06/00; «Ces tranquillisants impliqués dans les crimes», Le Parisien 07/02/02 ; «La drogue des violeurs en trois clics de souris» Le journal du dimanche 10/02/02 ; «La drogue des violeurs fait des ravages", Le Parisien, 01/07/02 ; «Alerte à la "drogue des violeurs"», Le Figaro 12/07/02 ; «Le GHB, drogue des violeurs», L'Express, 22/08/02.

Le nombre de cas de soumission chimique suspectée relevés aux UMJ en région Parisienne serait d'environ 20 par mois et nous pensons que ce nombre est probablement très inférieur à la réalité, car dans les conditions d'un viol sous soumission chimique, il est très difficile à une personne sur le plan psychologique d'aller porter plainte auprès des O.P.J., puis d'aller ensuite se faire examiner aux UMJ, de subir interrogatoire, examen clinique et prélèvements alors qu'elle est le plus souvent très choquée psychologiquement, non seulement par l'acte lui-même non consenti, mais également suite à l'angoisse due à l'amnésie provoquée par les substances psychoactives utilisées par les violeurs. Cette amnésie des faits étant le gage pour le violeur d'une certaine impunité. Ceci est bien matérialisé par le fait que lorsque plusieurs personnes dans un groupe subissent une soumission chimique avec viol, seules certaines d'entre elles acceptent d'aller porter plainte.

Dans un soucis d'efficacité optimale, la $2^{\text {eme }}$ section du parquet de Paris (Division du droit pénal général et de l'action publique de proximité) a défini une stratégie afin de garantir le recueil d'informations utiles à la manifestation de la vérité, d'une part en sensibilisant les services de police au stade de la prise de plainte ; d'autre part en établissant (en liaison avec les professionnels compétents : cliniciens, analystes..) un modèle de réquisition d'expertise toxicologique. Ce modèle de réquisition toxicologique (figure 1) matérialise la nécessité de prendre connaissance des éléments de la procédure policière, de prendre connaissance du compte-rendu d'observation médicale réalisée aux UMJ, de prendre en charge dès le début les prélèvements sanguins et urinaires effectués aux UMJ et confie à l'expert toxicologue désigné les analyses toxicologiques des prélèvements biologiques précités ; cette réquisition mentionne également à l'expert désigné l'obligation de procéder lui-même à des prélèvements pileux sur la victime (qu'il convoquera ultérieurement) avec nécessité pour lui d'interpréter les résultats de ses ana- lyses dans le cadre de tous les éléments précités portés à sa connaissance.

Notre expérience nous a appris que dans les dossiers d'expertise judiciaire de soumission chimique, il est souvent difficile d'aboutir à la preuve scientifique par l'analyse toxicologique et que cela relève souvent de la «performance analytique» comme le montre les différents cas exposés ci-après choisis spécifiquement pour illustrer la difficulté du problème.

\section{Matériel et méthode}

\section{Réactifs et solvants}

Le méthanol, l'acétate d'éthyle, le dichlorométhane et l'acétonitrile RS Plus sont de qualité HPLC (Prolabo). L'acide sulfurique $0,1 \mathrm{~N}$ et l'acide formique $99 \% \mathrm{RPE}$ sont de qualité pour analyse (Carlo Erba). La zopiclone a été obtenue auprès des laboratoires Aventis ; le zolpidem auprès de la firme Sanofi ; la niaprazine auprès de la société Genopharm.

\section{Analyses toxicologiques}

Les échantillons étaient traités en première intention en analyse de routine suivant une recherche systématique des principales familles de toxiques par chromatogra-

\section{EXPERTISE TOXICOLOGIQUE}

\footnotetext{
1. Prendte connaissance des teléments de la procèdure jointe.

2. Prendre connaissance du compre-tendu d'observation médicale téalisçe aux UMJ sur ........ ninsi que des premiers résultats d'analyse d'orientalion inmunochimiques réalisếes en urgence à l'hôpital .........

3. Prendre en charge les prèlèvements de sang ct d'urine opérés, le cas échểant, par le service des LMJ sur .... ..... qui vous seront remis par les Officiers de Police Judiciaire sous forme de scellés ainsi que les copies de la procédure et du dossier d'observation médicale.

4. Préciser si les déclarations de ........ sont compatibles avec l'administration de produits à son insu.

5. Convoquer au Laboratoire ......... (adresse) et procéder ì des prélèvements pileux (cheveux ou polis pubiens) en quantite nécessairc et suffisantc à l'exécution de vos analyses.

6. Procéder a l'analyse des prélèvements pileux opérés sur ........ ct dire s'ils fon apparaître des traces de produits psychotropes, stupéfiants ou de substances medicamenteuses: cn preciser la nature, la concentration, les effets sur lo compontencnt, le caractère ponctucl ou régulier de la consommation.

7. En cas de positivité, préciser si les drogues concernées peuvent âtre dissoutes ou ecrasces, de telic façon qu'on ne puisse en détecter la présence dans un verre, une tasse (thé, café...), ou dans un aliment (pâtisserie etc...)

8. Taire toute observations utiles â la manifestation de la vếrité

9. Reconstituer et restituer les scellés si ceux-ci n'ont pas çé détruits pour les besoins do l'expertise.

10. Rendre compte en urgence des premicrs résultats.
}

Figure 1 : Modèle de réquisition aux fins d'expertise toxicologique du Parquet du Tribunal de Grande Instance de Paris (Division du droit pénal général et de l'action publique de proximité) dans les cas de soumission chimique. 
phie en phase gazeuse couplée à la spectrométrie de masse (CPG/SM) selon un gradient de température classique sur colonne CPsil-8-CB (Varian) et par chromatographie en phase liquide couplée à un détecteur à barrette de diodes (HPLC/BD) sur chaîne Alliance Waters selon une technique précédemment publiée (15). L'alcool était dosé par chromatographie en phase gazeuse à détection thermoionique couplée à un injecteur à espace de tête (HS-CPG/FID) par une méthode validée.

Des techniques spécifiques étaient employées pour la recherche de produits moins communs ou présents à de très faibles concentrations. Les cas traités nécessitaient des extractions liquide-liquide et l'utilisation de techniques analytiques suffisamment sensibles et spécifiques telles que la chromatographie gazeuse couplée à la spectrométrie de masse tandem (CPG/SM/SM) et la chromatographie liquide couplée à la spectrométrie de masse tandem (HPLC/SM/SM). Seul le LSD était extrait par immunoaffinité. Les différentes techniques spécifiques mises en ouvre dans les cas cités sont décrites ci-après.

\section{$\left.1^{\circ}\right)$ Détermination des alcaloüdes végétaux toxiques}

La détermination des alcaloïdes de plantes toxiques dans les milieux biologiques est menée selon une technique précédemment publiée par chromatographie en phase liquide couplée à la spectrométrie de masse (16).

\section{$2^{\circ}$ ) Détermination du LSD}

La détermination du LSD dans les phanères utilise une précédente publication permettant d'atteindre une limite de détection de l'ordre de $0,5 \mathrm{pg} / \mathrm{mg}$ (17). Ils sont ainsi analysés par chromatographie en phase gazeuse couplée à la spectrométrie de masse tandem de même que le sang et l'urine, extraits selon les recommandations du fabriquant sur colonnes ImmunElute (Microgenics, France).

\section{$\left.3^{\circ}\right)$ Détermination du $\mathrm{GHB}$}

Le GHB est identifié sans dérivation dans les résidus de boisson par chromatographie en phase gazeuse sur colonne légèrement polaire couplée à la spectrométrie de masse, et présente un temps de rétention d'environ 5 minutes.

Son extraction des fluides biologiques est effectuée sur $200 \mu \mathrm{l}$ d'urine ou de sang par extraction liquide-liquide à l'acétate d'éthyle acidifié par $50 \mu \mathrm{l}$ d'acide sulfurique 0,1 N froid et utilise le GHB-d6 (Promochem, France) comme étalon interne.

Les cheveux sont traités selon une technique à paraître (18).

\section{$4^{\circ}$ ) Détermination du 7-aminoflunitrazépam}

La détermination du 7-aminoflunitrazépam dans les fluides biologiques et les phanères est réalisée en CPG/SM/SM sur TSQ 7000 (ThermoFinnigan).

$2 \mathrm{ml}$ de sang et/ou d'urine sont tamponnés à $\mathrm{pH} 8,6 \mathrm{par}$ $2 \mathrm{ml}$ de tampon bicarbonate $0,2 \mathrm{M}$ auxquels sont ajoutés $20 \mu \mathrm{l}$ de 7 -aminoflunitrazépam-d7 à $1 \mu \mathrm{g} / \mathrm{ml}$ dans le méthanol préparé par dilution d'une solution mère commercialisée à $100 \mu \mathrm{g} / \mathrm{ml}$ (Promochem, France). L'échantillon après avoir été homogénéisé est centrifugé à $2000 \mathrm{trs} / \mathrm{min}$ afin d'obtenir un surnageant clair pour extraction en SPE.

Les cheveux sont décontaminés par trois bains successifs de dichlorométhane puis broyés finement au broyeur à boulets. A $100 \mathrm{mg}$ pesés dans un tube vissé sont ajoutés $50 \mathrm{pg} / \mathrm{mg}$ de 7 aminoflunitrazépam-d 7 . L'échantillon est hydrolysé une nuit dans $1,5 \mathrm{ml}$ d'acide chlorhydrique $0,1 \mathrm{~N}$ à $56^{\circ} \mathrm{C}$. Après neutralisation par $150 \mu \mathrm{l}$ de soude $1 \mathrm{M}$, l'échantillon est tamponné par $2 \mathrm{ml}$ de tampon bicarbonate $0,2 \mathrm{M} \mathrm{pH} 8,6$ puis centrifugé à $3000 \mathrm{trs} / \mathrm{min}$, le surnageant clair est extrait en SPE selon le même protocole que pour les fluides biologiques décrit ci-dessous. L'éluat des phanères est filtré sur filtre PTFE 0,2 $\mu \mathrm{m}$ (Alltech, France).

L'extraction SPE est réalisée de façon classique sur colonne C18 ISOLUTE $200 \mathrm{mg} / 3 \mathrm{ml}$ (Bios Analytique, France). Les colonnes sont conditionnées par $4 \mathrm{ml} \mathrm{de}$ méthanol puis $2 \mathrm{ml}$ de tampon bicarbonate $0,2 \mathrm{M} \mathrm{pH}$ 8,6. Le surnageant de l'échantillon est déposé en totalité puis la colonne rincée par $1 \mathrm{ml}$ d'eau pure puis $1 \mathrm{ml}$ d'eau /méthanol $(50 / 50, \mathrm{v} / \mathrm{v})$. L'élution est réalisée par 3 fois $750 \mu \mathrm{l}$ de méthanol acidifié à $0,5 \%$ en acide acétique. L'éluat est évaporé sous courant d'air à $50-60^{\circ} \mathrm{C}$ puis dérivé par $30 \mu \mathrm{l}$ d'acétate d'éthyle et $30 \mu \mathrm{l}$ de HFBA (HeptaFluoroButyric Anhydre - Régis, Bios Analytique, France) 30 minutes à $60^{\circ} \mathrm{C}$. L'extrait dérivé est ensuite évaporé à sec sous courant d'air et repris par $20 \mu \mathrm{l}$ d'acétate d'éthyle pour injection en $\mathrm{CPG} / \mathrm{SM} / \mathrm{SM}$ en ionisation chimique négative.

Le système est constitué d'une CPG HP 5890 Série II plus munie d'une colonne CP-Sil-8-CB $30 \mathrm{~m} \times 0,25$ mm i.d., 0,25 $\mu$ m e.f. (Chrompack). La programmation de température est de $80^{\circ} \mathrm{C}$ pendant 2 minutes suivie d'un gradient de $15^{\circ} \mathrm{C} /$ min jusqu'à $310^{\circ} \mathrm{C}$, la température finale est maintenue 6 minutes. Le spectromètre de masse est un Finnigan Mat TSQ 7000 de chez ThermoFinnigan. La détection se fait en mode ionisation chimique négative et la collision de filiation se fait à une pression d'argon de 1,8 mTorr pour une énergie de $-18 \mathrm{eV}$. L'acquisition se fait en mode SRM (Selected Reaction Monitoring) à $\mathrm{m} / \mathrm{z} 366$ et 386 ions fils du m/z 466 (7-aminoflunitrazépam-d7) et $\mathrm{m} / \mathrm{z} 362$ et 382 ions fils du m/z 459 (7-aminoflunitrazépam). La limite de 
détection obtenue est inférieure à $1 \mathrm{pg} / \mathrm{mg}$ alors qu'une consommation régulière révèle des taux de l'ordre de $50 \mathrm{pg} / \mathrm{mg}$.

\section{$5^{\circ}$ ) Détermination du clonazépam et du 7-amino- clonazépam}

La détermination du clonazépam et du 7-aminoclonazépam dans les fluides biologiques est réalisée en HPLC/SM/SM après extraction de $1 \mathrm{ml}$ sur Toxitube-A (Amilabo, France).

Le système est constitué d'une HPLC Surveyor (ThermoFinnigan) munie d'une colonne Uptisphère ODB $5 \mu 150 \times 2 \mathrm{~mm}$ (Interchim, France) thermostatée à $30^{\circ} \mathrm{C}$. Un gradient de phase mobile à $320 \mu \mathrm{l} / \mathrm{min}$ est utilisé pour la séparation chromatographique. Les conditions initiales sont de $85 \%$ de tampon formiate $2 \mathrm{mM} \mathrm{pH} 3$ et $15 \%$ d'acétonitrile maintenu 0,5 minutes puis augmenté à $90 \%$ d'acétonitrile en 20 minutes et maintenu 10 minutes. Le retour aux conditions initiales est réalisé en 0,5 minute et l'équilibration se fait en 10 minutes. La durée de l'analyse est de 40 minutes. La détection se fait en masse tandem sur un spectromètre de masse LCQ Duo à trappe ionique (ThermoFinnigan) muni d'une interface électrospray, en mode d'ionisation positif, et permet la recherche simultanée de 8 benzodiazépines par injection, incluant l'étalon interne. L'acquisition utilise le scan complet des ions fils dont les majoritaires servant à la quantification sont à $\mathrm{m} / \mathrm{z}$ 255,1 et 274 ions fils du $\mathrm{m} / \mathrm{z} 320,2$ (clonazépam-d4, Promochem) pour une énergie de fragmentation de $40 \% ; \mathrm{m} / \mathrm{z} 251,1$ et 270 ions fils du m/z 316,1 (clonazépam) pour une énergie de fragmentation de $40 \%$ et $\mathrm{m} / \mathrm{z} 250$ ion fils du 286,2 (7-aminoclonazépam) pour une énergie de fragmentation de $32 \%$. L'ajout d'étalon interne se fait à $10 \mathrm{ng} / \mathrm{ml}$ de sang ou d'urine pour une limite de détection d'environ $1 \mathrm{ng} / \mathrm{ml}$.

\section{$\left.6^{\circ}\right)$ Détermination de la zopiclone}

La zopiclone est mise en évidence dans le sang, l'urine et les phanères par HPLC/SM/SM sur LCQ Duo à trappe d'ions.

L'extraction dans les fluides biologiques se fait en Toxitube-A utilisant la niaprazine à $10 \mathrm{ng} / \mathrm{ml}$ comme étalon interne et l'extrait est évaporé doucement sous courant d'air puis rapidement repris pour l'injection par $30 \mu \mathrm{l}$ d'acétonitrile.

L'extraction des phanères est réalisée sur une pesée de $50 \mathrm{mg}$ dans un tube vissé additionné de $1 \mathrm{ng} / \mathrm{mg}$ de niaprazine (Etalon interne) puis $2 \mathrm{ml}$ de tampon Soerensen $\mathrm{pH} 7,6$. Après sonication pendant une heure, une extraction liquide-liquide est réalisée par $2 \mathrm{ml}$ de dichlorométhane. Après centrifugation, la phase organique est filtrée sur filtre PTFE $1 \mu \mathrm{m}$ (Alltech, France) puis évaporée à froid et reprise par $30 \mu \mathrm{l}$ d'acétonitrile. $10 \mu \mathrm{l}$ des extraits sont injectés dans le système chromatographique. Le système est constitué d'une HPLC Surveyor (ThermoFinnigan) munie d'une colonne Kromasil C18 $5 \mu 150 \times 1 \mathrm{~mm}$ (Alltech, France) thermostatée à $30^{\circ} \mathrm{C}$. La phase mobile est délivrée de façon isocratique à $100 \mu \mathrm{l} / \mathrm{min}$ pour $10 \%$ de tampon formiate $5 \mathrm{mM} \mathrm{pH} 3$ et $90 \%$ d'acétonitrile maintenu 10 minutes. La durée de l'analyse est de 10 minutes. La détection se fait en masse tandem sur un spectromètre de masse LCQ Duo à trappe ionique (ThermoFinnigan) muni d'une interface électrospray. La détection se fait en mode d'ionisation positif et permet la recherche simultanée de zopiclone, zolpidem et niaprazine (étalon interne).

L'acquisition se fait en mode tandem avec scan complet des ions fils sur la zopiclone et le zolpidem. La zopiclone étant instable dans la source, il est nécessaire d'utiliser comme ion parent son fragment stable à $\mathrm{m} / \mathrm{z}$ 245. Les ions de quantification sont à : $\mathrm{m} / \mathrm{z} 217$ ion fils $\mathrm{du} \mathrm{m} / \mathrm{z} 245$ (fragment de la zopiclone) pour une énergie de fragmentation de $34 \%$ et $\mathrm{m} / \mathrm{z} 263,2$ et 235,3 ions fils du $\mathrm{m} / \mathrm{z}$ 308,0 (zolpidem) pour une énergie de fragmentation de $36 \%$. La limite de détection atteinte est de l'ordre de $4 \mathrm{pg} / \mathrm{mg}$ dans les phanères.

\section{$\left.7^{\circ}\right)$ Détermination de la niaprazine}

L'extraction de la niaprazine est réalisée dans les différents milieux biologiques comme pour la zopiclone en remplaçant le dichlorométhane par l'éther pour l'extraction des phanères et utilise la lidocaine comme étalon interne. Sa détection est réalisée en HPLC/SM/SM sur LCQ Duo à trappe d'ions par double fragmentation avec collision induite dans la source à une énergie de $34 \%$ dont l'ion fragment majoritairement produit $\mathrm{m} / \mathrm{z}$ 177,1 est cassé à une énergie de fragmentation de $30 \%$. L'acquisition se fait en mode scan complet des ions fils avec pour ion de quantification l'ion majoritaire à $\mathrm{m} / \mathrm{z}$ 148.9. La limite de détection obtenue est inférieure au nanogramme par milligramme dans les phanères.

\section{Résultats - Cas médicaux légaux}

\section{CAS n ${ }^{\circ} 1$ : Datura (Atropine-Scopolamine) : soumission chimique et meurtre d'enfants}

Deux scurs jumelles âgées de 7 ans et souffrant de problèmes psychomoteurs depuis leur enfance sont retrouvées brûlées dans leur habitation, comme endormies, sans signes de tentative de fuite. L'autopsie montrait des signes de violences ainsi que des résidus de fumées dans les trachées des deux fillettes. Des prélèvements de sang, cheveux et contenu gastrique étaient effectués. Une analyse de première intention révélait la présence de toxiques dus à l'incendie ( $\mathrm{HbCO}$, cyanures) et un 
traitement antiépileptique (acide valproïque, clonazépam, carbamazépine) qui n'expliquaient pas à eux seuls l'absence de réaction des fillettes face au feu. Des analyses complémentaires étaient donc réalisées pour la recherche des toxiques végétaux. (Tableau I).

Un screening d'alcaloïdes végétaux toxiques en LC/MS révélait la présence d'atropine et de scopolamine dans le sang et dans le contenu gastrique à des taux certes non mortels, mais suffisants pour que l'effet narcotique du Datura (hallucinogène narcotique incapacitant) les mettent dans un état de stupeur semi comateux pouvant expliquer leur incapacité de fuir. L'enquête a finalement déterminé l'incendie comme étant d'origine criminelle et a permis de trouver au domicile familial des ouvrages sur les plantes toxiques. $\mathrm{Au} \mathrm{vu}$ des résultats d'analyses toxicologiques, les parents ont finalement avoué avoir drogué leurs filles avec une infusion de feuilles de Datura, plante fréquente dans cette région, puis les avoir tuées par le feu, après que leur requête au bout de plusieurs années d'admission de celles-ci dans un institut spécialisé ait été refusée la veille.

Tableau I : Résultats d'analyse toxicologique dans le sang.

\begin{tabular}{|c|c|}
\hline Eilléte 1 & Eillette 2 \\
\hline HbCO : $11,3 \%$ & HbCO $: 11,9 \%$ \\
Acide valprö̈que $: 14,4 \mu \mathrm{g} / \mathrm{ml}$ & Acide valpröque $: 8,3 \mu \mathrm{g} / \mathrm{ml}$ \\
Clonazépam $: 0,9 \mu \mathrm{g} / \mathrm{ml}$ & Clonazépam $: 1,9 \mu \mathrm{g} / \mathrm{ml}$ \\
Cyanure $: 2,43 \mu \mathrm{g} / \mathrm{ml}$ & Cyanure $: 0,7 \mu \mathrm{g} / \mathrm{ml}$ \\
Atropine $: 32,5 \mathrm{ng} / \mathrm{ml}$ & Atropine $: 7,5 \mathrm{ng} / \mathrm{ml}$ \\
Scopolamine $: 4,4 \mathrm{ng} / \mathrm{ml}$ & Scopolamine $: 0,8 \mathrm{ng} / \mathrm{ml}$ \\
\hline
\end{tabular}

\section{CAS n ${ }^{\circ} 2$ : LSD : violence et viol en réunion}

Une jeune femme se présentait aux UMJ de Paris après avoir été violée dans un appartement. Invitée à boire un café chez une de ses connaissances, elle se retrouvait en compagnie de 3 autres jeunes garçons. Elle y boit un café et se trouve, un quart d'heure plus tard, prise de frissons et de malaise. Elle ressent des vertiges ainsi qu'une alternance d'hypothermie et d'hyperthermie et des visions de flash colorés.

Elle subit alors des violences sexuelles, physiques (cheveux arrachés) et morales par les quatre personnes présentes. Elle ne se souvient que partiellement des faits, et l'état de confusion dans lequel elle se trouvait ne lui a pas permis de résister à ses agresseurs.

Les premières analyses réalisées en immuno-chimie à l'hôpital s'avéraient négatives. Les analyses réalisées au laboratoire Toxlab révélaient outre la présence d'ibuprofène à taux infrathérapeutique, la présence de LSD dans le sang à $0,45 \mathrm{ng} / \mathrm{ml}$, dans l'urine à $0,12 \mathrm{ng} / \mathrm{ml}(\mathrm{LD}=0,05 \mathrm{ng} / \mathrm{ml})$ et son absence dans les phanères ( $\mathrm{LD}<0,5 \mathrm{pg} / \mathrm{mg}$ ).

L'analyse des traces se trouvant dans la tasse saisie par les O.P.J. révélait la présence de LSD, de caféine et d'acide chlorogénique manifestant son utilisation pour administration de café drogué au LSD. Les quatre jeunes gens ont été interpellés en possession de buvards de LSD et de comprimés d'Exctasy et ont avoué avoir drogué la jeune fille à son insu par l'ajout de deux buvards de LSD dans son café.

\section{CAS n ${ }^{\circ} 3$ : GHB et viol entre Officiers de Police Judiciaire}

Une jeune femme fonctionnaire de Police de race noire se fait offrir un verre de champagne chez elle vers 22 heures par un de ses collègues de travail pour fêter un heureux évènement. Tandis qu'elle s'occupe de son tout jeune enfant, celui-ci sert deux coupes et insiste pour qu'elle boive la sienne «d'un trait». Elle sombre dans l'inconscience quelques minutes environ après avoir bu sa coupe et se réveille 2 heures plus tard seule dans son lit mais sans vêtement et sans aucun souvenir des faits si ce n'est d'avoir bu la coupe de champagne. L'analyses de ses urines prélevées 24 heures après les faits montre un taux de GHB physiologique dans l'urine $=0,24 \mu \mathrm{g} / \mathrm{ml}$ et l'absence de toute autre substance médicamenteuse ou stupéfiante (7). L'analyse du reste de la bouteille de champagne utilisé montre l'absence de GHB dans celle-ci et sa présence dans les résidus restants dans la coupe retrouvée cassée dans le vide ordure. L'analyse de ses phanères met en évidence une augmentation significative de la concentration en GHB sur le segment correspondant aux faits comparativement aux précédents et qui reste supérieure au segment proximal (racine). La présence de GHB dans le verre et les phanères malgré l'absence de GHB dans un prélèvement d'urine effectué trop tardivement (délai maximum de détection dans l'urine du GHB de 4 à 10 heures selon la dose $(6,12,13,8)$ est en accord avec une prise exogène de GHB et corrobore les faits.

L'agresseur a reconnu avoir acheté la veille des faits de 1 '«Ecstasy liquide» vendu en flacon de $10 \mathrm{ml}$ dans un Sex-shop du $18^{\mathrm{ime}}$ arrondissement de Paris et en avoir versé dans la coupe de la victime.

\section{CAS 4 : GHB et viol en réunion}

Une jeune fille à l'occasion d'une soiré chez une connaissance rencontre 4 jeunes gens et absorbe une boisson alcoolisée vers minuit et sombre dans l'incons- 
cience peu de temps après. Elle se fait violer par un à quatre militaires du contingent d'origine Calédonienne en permission en Métropole. La jeune fille se réveille dévêtue dans un lit le lendemain des faits dans un lieu inconnu sans aucun souvenir, si ce n'est l'absorption d'une boisson alcoolisée de type punch.

En l'absence de sang et d'urine disponibles aux UMJ de l'hôtel Dieu de Paris nous avons réalisé plusieurs semaines après les faits un prélèvement de cheveux et effectué entre autres l'analyse des phanères pour la recherche de GHB (14). La concentration dans le segment correspondant aux faits est de $5,5 \mathrm{ng} / \mathrm{mg}$, tandis que la concentration moyenne déterminée avant les faits dans les cheveux de cette personne est de $1,8 \mathrm{ng} / \mathrm{mg}$.

L'enquête a démontré ultérieurement que les individus s'étaient rendus dans un Sex-shop du $18^{\mathrm{ime}}$ arrondissement de Paris peu avant les faits pour y acheter de l' «Ecstasy liquide», nom de fantaisie donné au GHB, vendu en flacon de $10 \mathrm{ml}$.

\section{CAS 5 : Flunitrazépam : la soumission chimique, un moyen d'existence}

Un homme d'une cinquantaine d'années d'origine Asiatique est retrouvé mort un matin d'hiver sans portefeuille et sans papier sous une porte cochère; la nuit est très froide, il est légèrement vêtu (ne porte pas de veste) et est allongé, comme endormi, dans une impasse soumise à un courant d'air froid au sol. Il est transporté à l'hôpital dans un coma hypothermique $\left(29^{\circ} \mathrm{C}\right)$ et décède peu après.

L'autopsie met en évidence un décès par hypothermie suite à une exposition prolongée au froid mais ne révèle ni pathologie visible, ni lésions traumatiques viscérales; ce qui n'explique pas l'absence de réaction de la victime au froid.

L'analyse du sang révèle une alcoolémie à $0,81 \mathrm{~g} / \mathrm{l}$, la CPG/ECD (détecteur à capture d'électron) met en évidence du midazolam : $15 \mathrm{ng} / \mathrm{ml}$ (anesthésique dont l'enquête montrera qu'il a été administré par le SAMU en réanimation). L'analyse en CPG masse tandem permet de détecter quelques picogrammes par millilitre $(13 \mathrm{pg} / \mathrm{ml})$ de 7 -aminoflunitrazépam, métabolite du flunitrazépam, une benzodiazépine hypnotique (principe actif du ROHYPNOL(B).

Ce résultat d'analyse a permis aux enquêteurs d'élucider l'affaire : le flunitrazépam n'était pas prescrit à la victime. Le soir précédent, il buvait une bière en compagnie d'un autre homme dans un bar voisin du lieu de sa découverte. Après son décès, sa carte bleue était utilisée ce qui a permis d'identifier et d'interpeller l'auteur des faits grâce à la caméra vidéo d'un distributeur de billets de banque et d'identifier le bar du délit grâce au ticket retrouvé dans sa poche.

Finalement les enquêteurs ont recueilli les aveux de l'auteur : il avait administré à la victime un comprimé de ROHYPNOL(B) pour lui voler sa carte bancaire ; c'était la $40^{\text {eme }}$ personne qu'il dépouillait ainsi et la soumission chimique était son moyen d'existence depuis 10 ans.

\section{CAS n6 : Flunitrazépam : vol en réunion de trois jeunes hommes}

Trois jeunes hommes de 30 ans passent la nuit dans un bar, un inconnu les aborde et leur offre un verre ; après avoir consommé la boisson, ils deviennent amnésiques. Deux d'entre eux reprenaient conscience au matin vers 05 h00 et constataient la disparition de leurs cartes bancaires, d'une montre et d'un pull-over, tandis que le troisième se réveillait dans un véhicule des pompiers intervenus sur la voie publique où il avait été recueilli, le visage présentant plusieurs plaies saignantes. Les analyses toxicologiques ont montré la présence de 7 aminoflunitrazépam (le métabolite actif du flunitrazépam) dans le sang des trois victimes aux taux respectifs de $5,8 \mathrm{ng} / \mathrm{ml}, 6,2 \mathrm{ng} / \mathrm{ml}$ et $5,9 \mathrm{ng} / \mathrm{ml}$; ainsi que dans l'urine prélevée sur deux des victimes le lendemain des faits. De plus l'analyse segmentaire des cheveux de l'une des victimes (pour les deux autres le prélèvement de cheveux était pondéralement insuffisant) montre bien la présence de 7-aminoflunitrazépam (19 pg/mg sur une période de 1 semaine comprenant la date des faits) (figure 2) et son absence sur les segments antérieurs et postérieurs prouvant qu'il n'était pas traité par ce médicament. Ce cas montre qu'une prise unique de flunitrazépam est donc détectable dans les phanères et corrèle les travaux de A. Negrusz (20). Ces analyses ont permis de conclure à l'ingestion (à leur insu et dans le but de les dépouiller) d'un ou deux comprimés de flunitrazépam par chacune des victimes le soir des faits expliquant également l'amnésie dont ils étaient frappés depuis la consommation de la boisson.

\section{CAS n ${ }^{\circ}$ : Clonazépam, GHB et viol}

Une jeune femme se réveille vers $14 \mathrm{~h}$ nue et seule au domicile de l'homme venu dîner chez elle la veille. Angoissée, celle-ci se rend aux U.M.J. de Paris pour être examinée et faire des analyses afin de déterminer si elle a été droguée à son insu. Elle se souvient être entrée dans un «état second» la veille lors du dîner : confusion, somnolence, euphorie..., avoir été transportée en moto et avoir eu un rapport sexuel. Les analyses montraient dans le sang la présence de clonazépam $(0,05 \mu \mathrm{g} / \mathrm{ml}), 7$-amino-clonazépam $(0,03 \mu \mathrm{g} / \mathrm{ml})$ et 


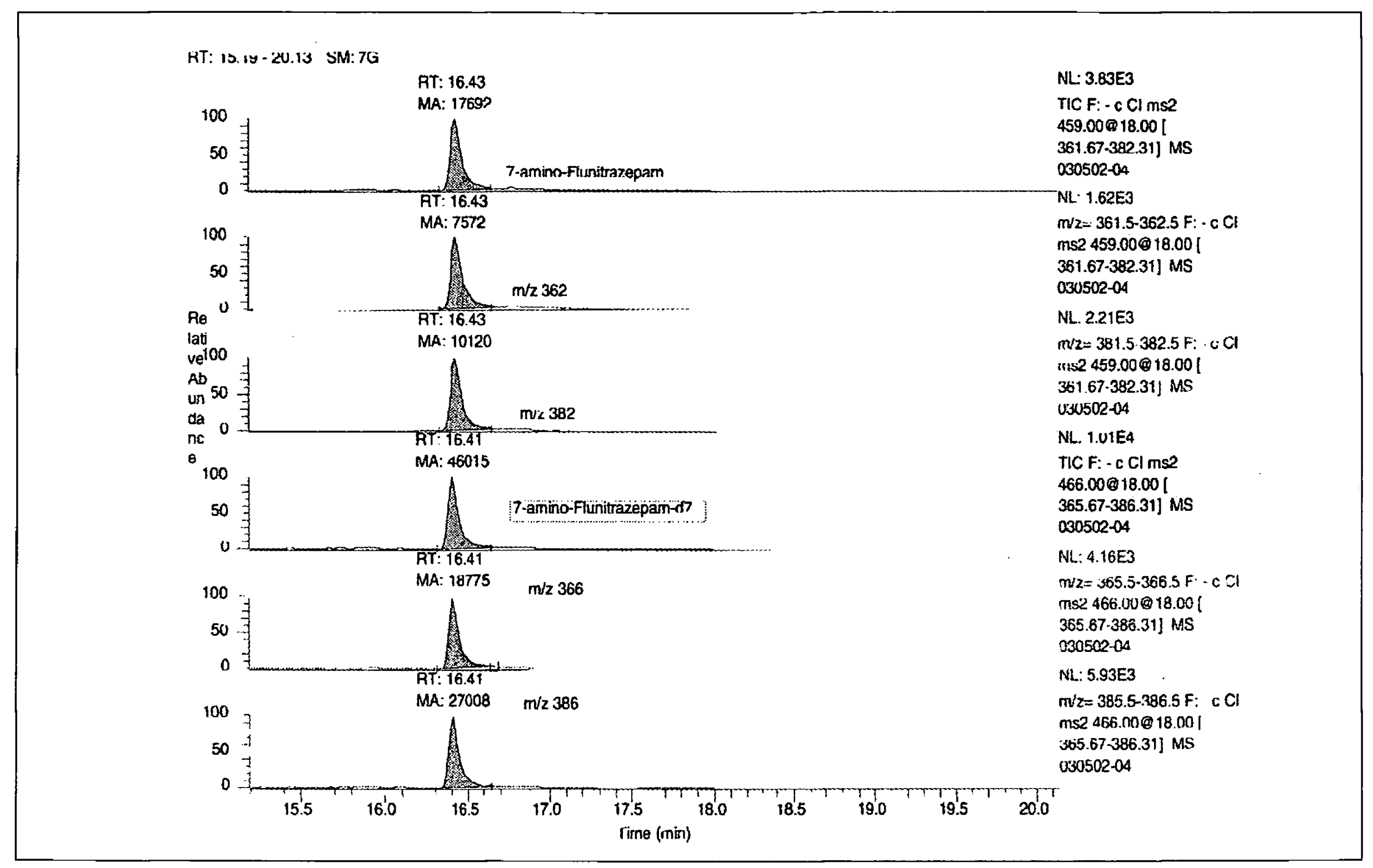

Figure 2 : Chromatogramme pour la détection du 7-aminoflunitrazépam ( $\mathrm{m} / \mathrm{z} 362$ et 382) à $19 \mathrm{pg} / \mathrm{mg}$ dans les cheveux de la victime avec pour étalon interne le 7-aminoflunitrazépam-d7 (m/z 366 et 386) en CPG-SM-SM et ionisation chimique négative.

pholcodine $(0,035 \mu \mathrm{g} / \mathrm{ml})$; dans l'urine : chlorphénamine, pholcodine $(2,5 \mu \mathrm{g} / \mathrm{ml})$ et 7 -amino-clonazépam $(0,65 \mu \mathrm{g} / \mathrm{ml})$. Deux verres utilisés durant le repas à son domicile étaient saisis par les O.P.J. et également analysés. Ils révélaient la présence de : gamma-hydroxybutyrate de sodium (GHB) et clonazépam dans l'un et gamma-hydroxybutyrate de sodium dans l'autre. Les prélèvements sanguins ont eu lieu $13 \mathrm{~h}$ à $18 \mathrm{~h}$ après les faits. Le gamma-hydroxybutyrate de sodium est rapidement éliminé de l'organisme, on estime que pour une prise deux fois supérieure à la posologie orale active classique soit $60 \mathrm{mg} / \mathrm{kg}$, le temps maximal de détection est dans le sang de 5 heures et dans l'urine de 5 à 12 heures $(7,11)$. Le GHB ne pouvait donc pas être mis en évidence dans le sang et l'urine, à l'inverse du clonazépam dont la demi-vie d'élimination varie de 20 à 60 heures permettant d'identifier cette molécule dans le sang bien que les prélèvements furent tardifs.

\section{CAS n ${ }^{\circ}$ : Vraie soumission chimique et faux résultat analytique : pièges à éviter}

Une femme âgée de 55 ans dîne avec un collègue de travail connu de longue date. Quasi-amnésique à son réveil vers 8 heures du matin à son domicile, elle se souvient avoir passé la nuit avec lui et pense avoir été droguée par cet homme. Des prélèvements sont effectués aux UMJ de Paris et une première analyse toxicologique est réalisée par un laboratoire hospitalier en CPG/SM annonçant la présence dans l'urine d'hydroxyzine (ATARAX®) et ses métabolites. Par ailleurs l'enquête indique une prise possible d'HALCION $\mathbb{B}$ (triazolam) retrouvé au domicile de son collègue mis en cause. La plaquette retrouvée chez lui met en évidence deux comprimés manquants.

Face à cette contradiction une contre-expertise des prélèvements est demandée par le Juge d'Instruction au toxicologue expert. Nos analyses n'ont pas permis de mettre en évidence le triazolam, ni son métabolite l'hydroxytriazolam dans l'urine prélevé à l'hôpital ni dans celle prélevée le matin même par la victime et soit disant mise à $+4^{\circ} \mathrm{C}$ dans son réfrigérateur. Une étude faite spécifiquement au laboratoire sur la stabilité de l'hydroxytriazolam dans l'urine révèle que celui-ci est très instable à $+4^{\circ} \mathrm{C}$. Le délai de 10 jours entre les faits et la réception des prélèvements au laboratoire permet parfaitement d'expliquer ce résultat négatif. On 
apprendra par la suite que l'urine collectée à 08 heures le matin des faits par la victime a été, en réalité, oubliée dans son sac et n'a été mise au frigidaire que 8 jours après.

Concernant la mise en évidence d'hydroxyzine par chromatographie en phase gazeuse couplée à la spectrométrie de masse lors de la première expertise sur les urines analysées à l'hôpital, nous avons observé les «pièges analytiques» suivants (figure 3):

- l'ion majoritaire $\mathrm{m} / \mathrm{z}=201$ de l'hydroxyzine est un ion non spécifique car il est aussi présent dans de nombreuses interactions liées à des effets de matrice or il n'y avait pas dans leur spectre d'autres ions de confirmation permettant de conclure à la présence d'hydroxyzine.

- Il convient de s'assurer que le temps de rétention du composé identifié est rigoureusement identique à celui d'une référence d'hydroxyzine

- l'ion moléculaire $\mathrm{m} / \mathrm{z}=374$ doit être nécessairement confirmé, lorsqu'il est peu abondant, par une méthode alternative mettant en jeu un mode d'ionisation qui n'est pas celui classiquement utilisé en spectrométrie de masse à savoir l'ionisation chimique en mode positif et non l'impact électronique, et ce pour une meilleure spécificité.

- Dans le cas de l'hydroxyzine qui est une molécule chlorée, on doit nécessairement confirmer la présence de l'atome de chlore qui peut être aisément identifié par la répartition isotopique particulière de cet halogène sur le couple chlore 35/37. Il s'ensuit une répartition d'un tiers (abondance naturelle du chlore 37 ) de la masse $\mathrm{m} / \mathrm{z}+2$ sur chaque ion du spectre de masse contenant cet atome de chlore.

Rappelons parmi les critères unanimement reconnus sur le plan scientifique qu'afin d'identifier une molécule de façon certaine en CPG/SM, il faut nécessairement $s$ 'assurer de trois critères :

- quatre ions, dont l'ion moléculaire lorsqu'il est présent, sont nécessaires pour l'identification,

- le rapport des principaux ions entre eux doit être respecté,

- le temps de rétention de la molécule doit être identique à la référence.

Au vu de ces éléments, les méthodes alternatives entre-

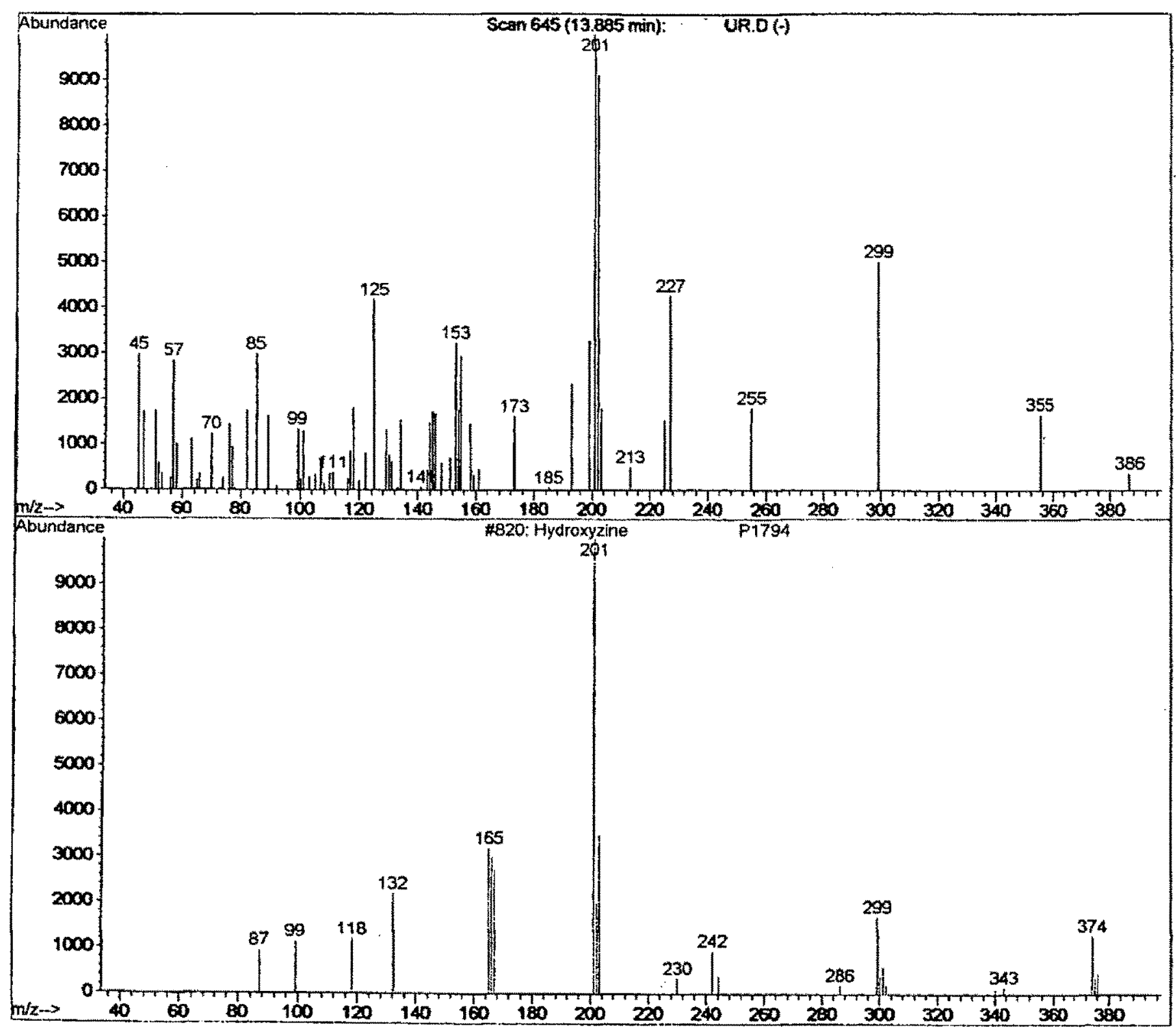

Figure 3 : Spectre de masse d'un faux positif à l'hydroxyzine dans une urine analysée en $C P G$ SM comparé au spectre de masse de référence de l'hydroxyzine. 
prises pour confirmer la présence de cette molécule, nous ont conduit à conclure à l'absence d'hydroxyzine et à confirmer la présence d'ions interférents par effet de matrice pouvant induire un résultat «faux positif».

\section{CAS n 9 : Niaprazine : Soumission chi- mique simultanée de 6 jeunes enfants}

Les parents de deux jeunes enfants ( 2 ans et 5 ans), confiés à une assistante maternelle dans la journée, constatent le soir chez leurs deux enfants des symptômes alarmants : somnolence anormale, yeux rougis, convulsions, réaction allergique allant jusqu'à l'œdème de Quincke. Une enquête est alors menée auprès de la nourrice qui avoue avoir en charge, simultanément 6 enfants âgés de 6 mois à 5 ans. Des expertises toxicologiques sont effectuées sur les cheveux des six enfants à la demande d'un magistrat instructeur. Les analyses de cheveux (sur une période allant de 1 mois et demi à 4 mois et demi selon la longueur des cheveux des enfants) ont révélés chez tous les enfants la présence de niaprazine principe actif du NOPRON®. Les taux retrouvés diffèrent selon que les enfants étaient confiés quotidiennement ou non à l'assistante maternelle $(4,12$ $\mathrm{ng} / \mathrm{mg}, 42,4 \mathrm{ng} / \mathrm{mg}, 46,9 \mathrm{ng} / \mathrm{mg}, 29,2 \mathrm{ng} / \mathrm{mg}, 8,55$ $\mathrm{ng} / \mathrm{mg}$ et $4,5 \mathrm{ng} / \mathrm{mg}$ ).

Le sirop NOPRON® est indiqué dans l'insomnie occasionnelle de l'enfant. Ce médicament doit être administré le soir au coucher et est réservé à l'enfant de plus de 3 ans, il est classé sur la liste II des substances vénéneuses depuis mars 2001 après avoir été auparavant en vente libre. La nourrice mise en examen a finalement avoué qu'elle administrait régulièrement aux enfants (dont certains très jeunes : 6 mois) du NOPRON® afin de les rendre moins turbulents voire somnolents, ne pouvant s'occuper des six enfants qu'elle avait accepté de garder simultanément.

\section{CAS n'10 : Zopiclone : viol et club de sport}

Une jeune femme de 28 ans rencontre un homme de 41 ans dans un club de sport. Celui-ci l'invite à boire un verre chez lui. Après avoir ingéré le cocktail servi par cet homme, la jeune femme déclarait n'avoir que des souvenirs très confus, qu'elle se remémorait uniquement par bribes et avoir manifestement subi une relation sexuelle non consentie à laquelle elle n'avait pu s'opposer en raison de son état de faiblesse consécutif à l'absorption de la boisson. La jeune femme ne se souvenait pas de quelle manière elle avait regagné son domicile et angoissée, elle portait plainte pour viol le lendemain en se rendant aux U.M.J. de Paris.
Consécutivement une information judiciaire était ouverte et des prélèvements de sang et d'urine ont été effectués. Les tests immunochimiques faits au laboratoire de l'hôpital étaient négatifs alors que nos analyses toxicologiques ont révélé dans l'urine la présence de zopiclone (TMOVANEß) au taux de $0.67 \mu \mathrm{g} / \mathrm{ml}$ et son absence du sang, expliquée par une demi-vie plasmatique de 5 heures. L'analyse des cheveux quant à elle a montré la présence de zopiclone à $13 \mathrm{pg} / \mathrm{mg}$ dans le segment correspondant à la période des faits (figure 4) et son absence dans les segments antérieurs et postérieurs aux faits. La zopiclone est un hypnotique qui entraine une forte altération de la vigilance et des réflexes pouvant conduire à l'endormissement et qui, hors du contexte ou il est habituellement prescrit (insomnie), peut produire une réelle soumission chimique.

\section{Conclusion}

- La mise en évidence de la soumission chimique par la preuve scientifique irréfutable obtenue grâce à l'expertise toxicologique, nécessite une collaboration étroite entre l'officier de Police Judiciaire, le médecin clinicien des Urgences Médico-Judiciaires, l'expert toxicologue et bien entendu la personne soumise chimiquement. Ainsi, dans certains des cas cités, on remarque que la preuve formelle est apportée, par le toxicologue analyste, grâce à l'analyse de traces dans une tasse à café ou sur des verres brisés, traces absentes des prélèvements biologiques.

- Par suite du délai souvent important entre la prise du produit et les faibles taux de substance active à rechercher, la détection dans les prélèvements de très faibles quantités des substances utilisées dans la soumission chimique nécessite des moyens analytiques importants (Chromatographie en Phase Gazeuse/Spectrométrie de Masse, Chromatographie Liquide Haute performance/Spectrométrie de Masse, Chromatographie en Phase Gazeuse/Masse tandem, Chromatographie Liquide Haute performance/Masse tandem). Le fait d'effectuer soi-même le prélèvement de cheveux et de connaître les caractéristiques du tableau clinique de la soumission chimique en questionnant la victime, permet au toxicologue d'orienter spécifiquement son analyse et donc, les moyens d'extraction et de détection à mettre en ceuvre. En effet la soumission chimique à la suite d'une prise d'alcool abusive, d'une consommation de cannabis exagérée ou fortement titrée, d'une prise de benzodiazépines ou apparentée à demi-vie d'élimination courte (triazolam, flunitrazépam, zopiclone) ou encore d'une prise de GHB ne procurent pas le même tableau clinique. Les caractéristiques de l'amnésie, sa 
durée, le réveil, la somnolence après réveil, les vomissements éventuels sont des éléments qui peuvent servir à orienter vers la famille chimique du produit administré.

- L'analyse des cheveux est très fréquemment capitale pour apporter la preuve de la prise de l'agent chimique au moment des faits ainsi que son abstinence avant et après les faits. La mise en évidence, par le toxicologue analyste, de la molécule chimique utilisée par le violeur présumé dans les prélèvements de la victime, permet aux enquêteurs de rechercher le produit dans l'environnement du mis en cause voire sur lui-même ; car le plus souvent, celui-ci, du fait de l'amnésie et devant l'impossibilité qu'ont habituellement les laboratoires à détecter ces molécules, se croit hors d'atteinte. L'aveu s'en suit assez souvent au vu d'un résultat toxicologique probant et inattendu.

- Face à la complexité du problème analytique, matérialisée par les cas précités, il est bien évident que l'utilisation des tests immunochimiques urinaires est ineffi- cace $(21,22)$, voire nuisible par leur négativité apparente, que même la chromatographie gazeuse couplée à la spectrométrie de masse utilisée dans certains laboratoires hospitaliers est insuffisante de même que la chromatographie liquide haute performance couplée à la barrette de diodes et qu'il est donc impératif de pouvoir également disposer de Chromatographie en Phase Gazeuse/Masse tandem/en Impact Electronique et Ionisation Chimique positive et négative et de Chromatographie en Phase Liquide/Masse tandem.

La soumission chimique étant un acte criminel, il nous semblerait logique, qu'à l'image de ce qui a été réalisé pour les empreintes génétiques, le Ministère de la Justice en liaison avec le Ministère de la Santé agréent au niveau national quelques laboratoires spécialisés. Cet agrément serait donné après épreuve qualifiante, à des experts toxicologues particulièrement compétents y compris dans l'analyse des cheveux dont on a vu le rôle capital dans les exemples ci-dessus et obligatoirement équipés de l'ensemble du matériel indispensable précédemment cité.

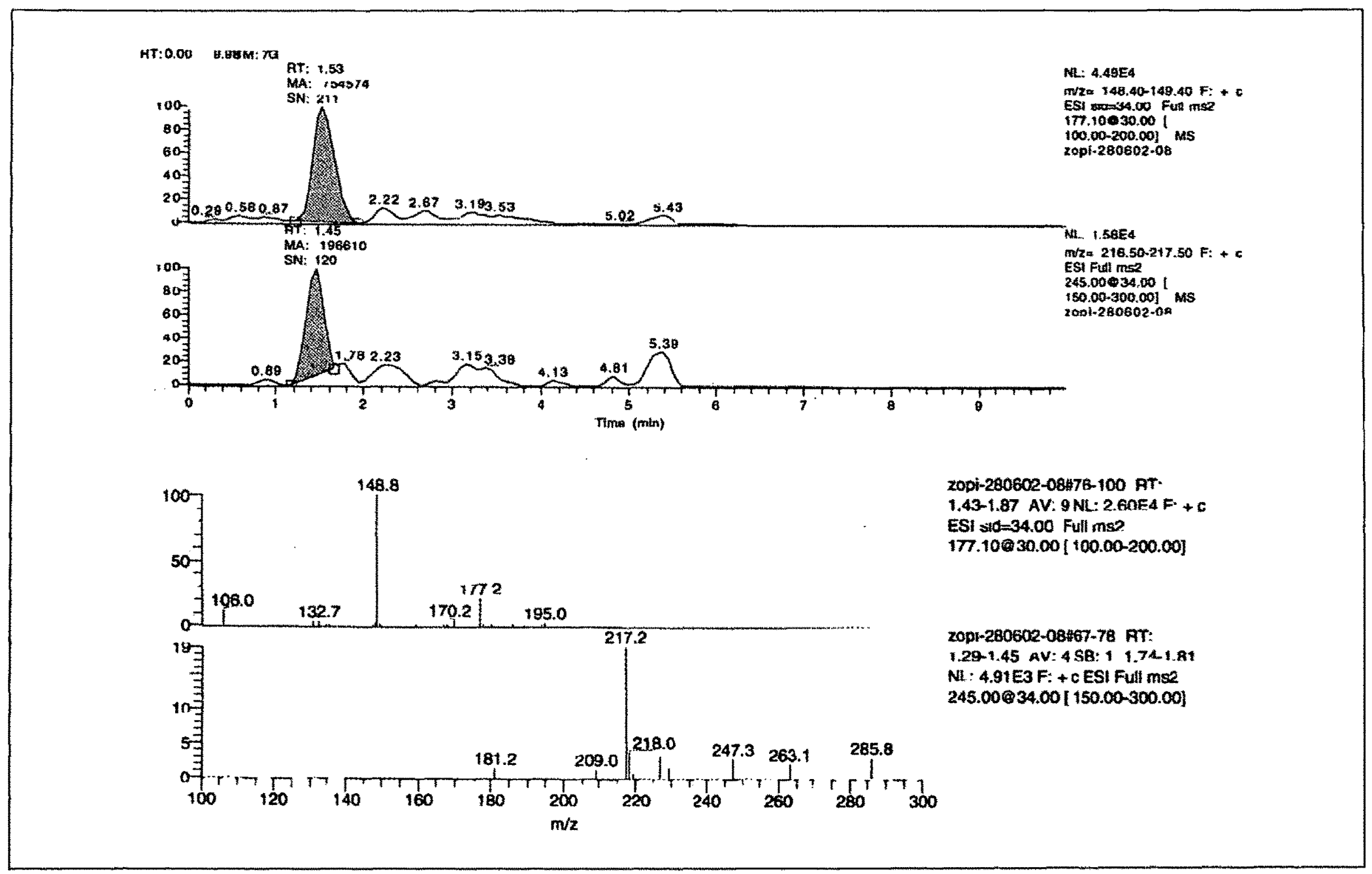

Figure $4:$ Chromatogramme et spectres de masses pour la détection de la zopiclone ( $\mathrm{m} / 2$ 217) à $13 \mathrm{pg} / \mathrm{mg}$ dans les cheveux de la victime avec pour étalon interne la niaprazine $(\mathrm{m} / \mathrm{z}$ 148.8) en HPLC-SM-SM à trappe d'ions et source électrospray. 


\section{Références}

1. ElSohly M.A., Salamone S.J. Prevalence of drugs used in cases of alleged sexual assault. J. Anal. Toxicol. 1999 ; $23: 141-146$.

2. Deveaux M., Renet S., Renet V., Gaulier JM., Kintz P., Verstraete A., Gosset D. Utilisation de l'acide gammahydroxybutyrique (GHB) dans les rave-parties et pour la soumission chimique en France : mythe ou réalité ? Acta Clinica Belgica 2002 ; 57 supp. 1 : 37-40.

3. Djezzar S., Dally S., Utilisation détournée et soumission médicamenteuse : le gamma-OH en question. Méd. Lég. Hospitalière. $1999 ; 2: 37$.

4. Questel F., Bécour B., Dupeyron J.P. ; Gailliot-Guilley M., Diamant-Berger O. Soumission médicamenteuse : 4 ans d'expérience aux UMJ de Paris. J. Méd. Lég. Droit Médical. $2000 ; 43: 459-465$.

5. Raul J.S., Kintz P., Géraut A., Tracqui A., Ludes B. La soumission chimique dans les agressions sexuelles. Prise en charge médico-légale à Strasbourg. J. Méd. Lég. Droit Médical. $2002 ; 45: 155-157$.

6. Kintz P., Villain M., Cirimele V., Goullé J.P., Ludes B. Usage criminel de substances psychoactives: le problème de la durée de détection. Acta Clinica Belgica. 2002 ; 57 supp. $1: 24-3$.

7. Gaillard Y., Goullé J.P., Pépin G. Le GHB dans la soumission chimique, en médecine légale et en thérapeutique : Interprétation des résultats : sang, urine, cheveux. La Clusaz 2001 : Résumé : $9^{\text {ime }}$ Congrès annuel de la Société Française de Toxicologie Analytique.

8. Verstraete A. Fenêtre de détection des xénobiotiques. $40^{\text {ine }}$ congrès de toxicologie clinique, 24-27 mars 2002, Djerba.

9. Ghysel M.H., Pépin G., Kintz P. La soumission médicamenteuse. Toxicorama. $1998 ; 10: 126-127$.

10. Goullé J.P., Kintz P. Un nouveau moyen d'investigation biologique : l'analyse des cheveux. Intérêt en pratique médicale. Rev. Med. Interne. 1996 ; 17 : 826-835.

11. Kintz P. Difficultés analytiques et d'interprétation dans les expertises toxicologiques liées à la présence de GHB. J. Med. Leg. Doit Médical. 2002 ; sous presse.

12. Ferrara S.D., Zotti S., Tedeschi L. et al. Pharmacokinetics of gamma-hydroxybutyric acid in alcohol dependant patients after single and repeated oral doses. Br. J. Clin. Pharmacol. $1992 ; 45: 353-356$.
13. Kintz P., Goullé J.P., Cirimele V., Ludes B. Window of detection of GHB in blood and saliva. Clin. Chem. $2001 ; 47: 2033-2034$.

14. Kintz P., Cirimele V., Jamey C., Ludes B. Lettre à la rédaction : soumission chimique par GHB : cheveux et GC/MS/MS. A.T.A. $2002 ; 14: 129-131$.

15. Gaillard Y., Pépin G. Use of high-performance liquid chromatography with photodiodes-array UV detection for the creation of a 600 -compound library. Application to forensic toxicology. J ; Chromatogr. A. $1997 ; 763$ : 149-163.

16. Cheze M., Gaillard Y., Pépin G. Réponses analytiques aux intoxications par substances végétales. A.T.A. 2000 ; $12: 307-314$.

17. Cheze M., Vayssette F, Pépin G. Dosage du LSD dans les phanères par chromatographie liquide couplée à la spec. trométrie de masse ou par chromatographie gazeuse couplée à la spectrométrie de masse tandem. A.T.A. 2001 ; $13: 63-68$.

18. Goullé J.P., Pépin G., Chèze M. Determination of endogenous levels of GHB in human hair. Are there possibilities for the identification of GHB administration through hair analysis in cases of drug-facilitated sexual assault ? J. Anal. Toxicol. Soumis au JAT en août 2002.

19. Ghysel M-H. Le GHB : l'acide gamma-hydroxybutyrique. Revue de la littérature. Toxicorama. $1999 ; 11: 1$ 11.

20.Negrusz A., Moore C.M., Hinkel K.B., et al. Deposition of 7-aminoflunitrazepam and flunitrazepam in hair after single dose of Rohynol. J. Forensic Sci. 2001 ; 46 : 1143 115.

21. Seigle-ferrand B, Dupeyron J.P., Papin-Castela N., Lacroix C., Goullé J.P., Chast F. Improvement of standard immunoassay for benzodiazepine trace detection in urine. Ther Drug Monit. 1999 ; 21 : Abstract : 6th International congress of TDM-CT.

22. Vasseur P. Agression sexuelle après soumission médicamenteuse. Med. Leg. Hospit. $2000 ; 3: 94$. 